\title{
Strobilanthes Kunthianus: An Overview
}

\author{
Ayishath Shabna, Syed Asadulla, Dr. Ajith Babu T K, and Dias Dileep E \\ Malik Deenar College of Pharmacy
}

\begin{abstract}
The use of herbal drugs in developing newer drug technology has increased to a greater extent. Strobilanthes species are widely spread in Asia and are endemic to Western Ghats India. They are known to have many traditional uses such as anti-inflammatory, anti-oxidant, anti-microbial, antiosteoarthritics etc. Strobilanthes kunthianus is widely distributed in the grassland of Nilgiri hills and blooms once in 14 years and they are also known to have many traditional uses. This review focuses on basic studies such as phytochemical screening and pharmacological effect of plant.
\end{abstract}

Keywords: Strobilanthes kunthiana, anti-inflammatory, antioxidant, anti-microbial, phytochemical screening

\section{INTRODUCTION}

$\mathrm{H}$ erbal drugs are used widely in newer drug development technology. The huge structural variety, advanced techniques for isolation, characterisation of the structure have led to the synthesis of drugs from plants. ${ }^{(1)}$ Nowadays plantbased drug development has dominated synthetic drug development to an instance. Isolation of medicinal compounds from the plant has greater influence and led to the development of newer drugs and modern therapies.

Strobilanthes kunthianus is widely distributed in the grassland of Western Ghats of India and blooms every 12 years. The name 'blue mountain' which is the synonym of Nilgiri hills is due to the presence of purplish flowers of S.kunthianus Nilgiri itself has 150 species of Strobilanthes. ${ }^{(2)}$ Strobilanthes species are known to have many medicinal properties such as antiinflammatory, anti-osteoarthritic, analgesic, anti-oxidant, hepatoprotective, anti-fungal, anti-giardial, and anti-microbial and protection against UV rays. ${ }^{(3)}$

\section{SYNONYM ${ }^{(3,4)}$}

Phleophyllum kunthianus

Strobilanthes kunthianus

Strobilanthes nilgirianthsis

Ruellia kunthianus

$$
\text { III. COMMON NAME }{ }^{(4)}
$$

Tamil: neelakurinji

Malayalam: neelakurinji

Hindi: kurinji

$$
\text { IV. TAXONOMIC TREE }{ }^{(4)}
$$

Kingdom: Plantae

Sub-kingdom: Phanerogamia

Division: Angiospermia

Class: Eudicots

Sub-class: Asterids
Order: Lamiales

Family: Acanthaceae

Genus: Strobilanthes

Botanical name: Strobilanthes kunthianus Nees T Anderson

\section{DISTRIBUTION ${ }^{(4)}$}

Found in tropical south and south East Asia, more than 300 species in Asian countries

Endemic to western ghats, India: Kerala, Tamil nadu \& Karnataka

\section{DESCRIPTION ${ }^{(3)}$}

It is a small shrub of height $30-60 \mathrm{~cm} \&$ in some conditions may grow upto $2 \mathrm{~m}$

Stem: numerous, erect, prominent nodes, quadrangular

Leaves: elliptic-ovate, $5 \times 2.5 \mathrm{~cm}$, acute at apex and base, margin-crenate-serrate, secondary veins upto 9 pairs, petioles $5 \mathrm{~mm}$ long

Inflorescences: spike, $8 \mathrm{~cm}$ long, uninterrupted, branched sometimes, support by leaf bract

Flower: corolla-tubular ventricose expanding at the base, inside hairy, 5-lobes, 2 -stamens, $7 \mathrm{~mm}$ long filament, ovaryhairy at tip \& glaborous, style-length $1.5 \mathrm{~cm}$, capsule $-1.2 \mathrm{~cm}$ long, oblong, 4 seeded

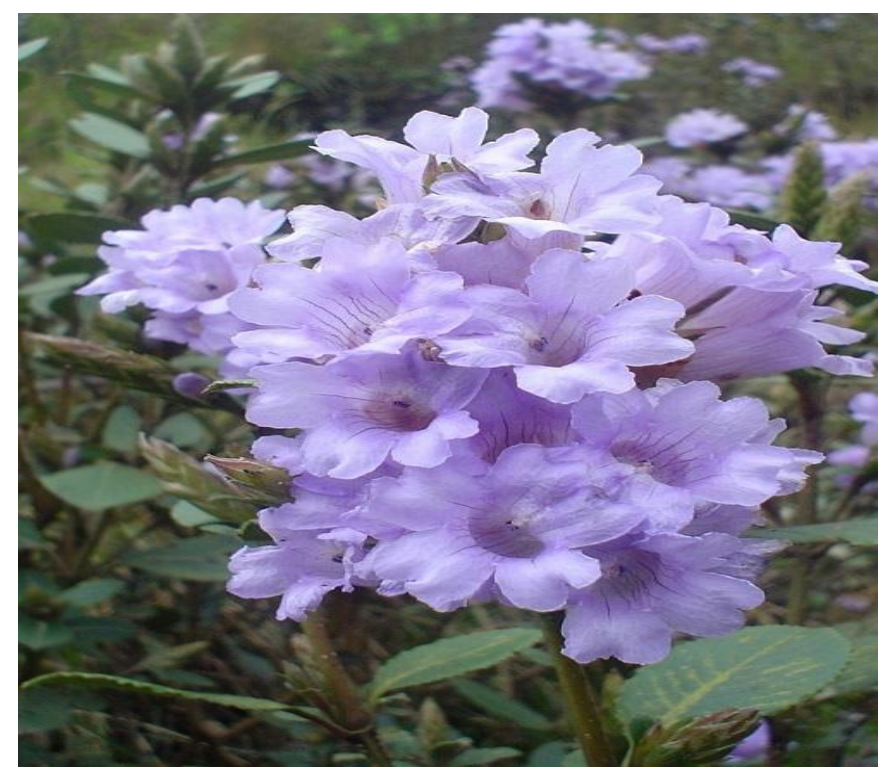

Figure 1: Strobilanthes kunthianus

$$
\text { VII. PHYTOCHEMICAL PROFILE }{ }^{(3)}
$$

The preliminary phytochemical screening by successive solvent extraction was made on stem and root. Solvents used 
were Pet. ether, chloroform, ethyl acetate \& methanol. Phenolic compound, steroids, and triterpenoids were present in pet. ether and chloroform extract of root and stem. Successive methanolic and ethyl acetate extract of root contained flavonoids, phenolics \& glycosides, while in the stem it showed phenolics, glycosides \& saponins.

\section{PHARMACOLOGICAL EFFECT}

\subsection{Anti-Inflammatory Activity ${ }^{(2)}$}

\subsubsection{Carrageenan induced paw edema}

In carrageenan-induced paw edema initial volume of the hind paw of the rat was calculated with a plethysmometer. The rat was grouped into control \& test groups. The control gets the only vehicle. Here, carrageenan in saline $(1 \% 0.1 \mathrm{ml} / \mathrm{rat})$ was injected subcutaneously on the right hind paw. The test group was treated with methanolic extract of flowers, stems, roots and leaves. At an interval of $1 \mathrm{~h}, 2 \mathrm{~h} \& 3 \mathrm{~h}$ the paw volume was measured \& percentage increase in the volume of paw between test and percentage inhibition was found to be increasing with time and methanolic flower extract has shown percentage inhibition of $21.70 \pm 1.17$ to $40.50 \pm 0.36$ $\& 30.82 \pm 0.63$ to $50.76 \pm 0.66$ at dose $100 \& 200 \mathrm{mg} / \mathrm{kg}$ at $1-3$ hour interval. Ibuprofen was used as standard.

\begin{tabular}{|c|c|c|c|c|}
\hline \multirow{2}{*}{ Treatment } & $\begin{array}{c}\text { Dose } \\
(\mathrm{Mg} / \mathrm{kg})\end{array}$ & $1 \mathrm{~h}$ & $2 \mathrm{~h}$ & $3 \mathrm{~h}$ \\
\cline { 3 - 5 } & - & - & - & - \\
\hline Control & & 4 inhibition & \%inhibition & \%inhibition \\
\hline Ibuprofen & 100 & $43.79 \pm 1.71$ & $65.62 \pm 0.81$ & $71.69 \pm 0.84$ \\
\hline $\begin{array}{c}\text { Crude } \\
\text { flower } \\
\text { extract }\end{array}$ & 100 & $24.55 \pm 1.50$ & $38.10 \pm 0.72$ & $42.70 \pm 0.82$ \\
\cline { 2 - 5 } & 200 & $45.50 \pm 1.25$ & $56.30 \pm 0.84$ & $58.90 \pm 0.94$ \\
\hline \multirow{2}{*}{$\begin{array}{c}\text { Crude root } \\
\text { extract }\end{array}$} & 100 & $21.10 \pm 1.70$ & $27.27 \pm 0.36$ & $38.59 \pm 1.14$ \\
\cline { 2 - 5 } & 200 & $33.43 \pm 1.70$ & $42.32 \pm 1.02$ & $47.04 \pm 0.46$ \\
\hline $\begin{array}{c}\text { Crude } \\
\text { stem } \\
\text { extract }\end{array}$ & 100 & $20.61 \pm 1.41$ & $24.43 \pm 0.57$ & $31.74 \pm 1.08$ \\
\cline { 2 - 5 } & 200 & $35.41 \pm 2.69$ & $34.37 \pm 0.73$ & $38.59 \pm 0.82$ \\
\hline $\begin{array}{c}\text { Crude } \\
\text { leaves } \\
\text { extract }\end{array}$ & 100 & $16.67 \pm 1.78$ & $19.88 \pm 1.46$ & $29.91 \pm 0.65$ \\
\cline { 2 - 5 } & 200 & $26.53 \pm 0.91$ & $30.39 \pm 0.81$ & $42.24 \pm 0.65$ \\
\hline
\end{tabular}

Table: 1 Anti-inflammatory activity of crude methanolic extract of S.kunthianus in carrageenan induced paw edema

\subsection{Analgesic Activity ${ }^{(2)}$}

\subsubsection{Hot Plate Method}

Eddy's hot plate method was used. The temperature was set at $55 \pm 0.5^{\circ} \mathrm{c} \&$ treated rats were positioned on a plate. The reaction time of rats to respond to heat was noted. A response may be paw licking, jumping, or paw withdrawing. This was tested at $0,30,60,90 \& 120 \mathrm{~min}$ after treating with extract orally. Methanolic extract of leaf, flower, stem, and root was used among which methanolic flower extract
Shown more potent activity. The percentage protection was 60.96 to $173.10 \%$ at 30 to 90 minutes at dose $100 \mathrm{mg} / \mathrm{kg} \&$ 112.92 to $233.3 \%$ at $200 \mathrm{mg} / \mathrm{kg}$

\begin{tabular}{|c|c|c|c|c|c|c|}
\hline & \multirow{2}{*}{$\begin{array}{c}\text { Dose } \\
\text { Treatment }\end{array}$} & \multicolumn{5}{|c|}{ \%potency } \\
\cline { 3 - 7 } & $\mathrm{Mg} / \mathrm{kg})$ & $0 \mathrm{~min}$ & $\begin{array}{c}30 \\
\text { min }\end{array}$ & $\begin{array}{c}60 \\
\text { min }\end{array}$ & $\begin{array}{c}90 \\
\text { min }\end{array}$ & $\begin{array}{c}120 \\
\text { min }\end{array}$ \\
\hline Control & - & - & - & - & - & - \\
\hline Ibuprofen & 100 & 0 & 199.43 & 242.43 & 287.43 & 272.95 \\
\hline $\begin{array}{c}\text { Crude } \\
\text { flower } \\
\text { extract }\end{array}$ & 100 & 0 & 60.96 & 135.59 & 173.10 & 169.40 \\
\cline { 2 - 7 } & 200 & 0 & 112.92 & 194.07 & 233.30 & 220.77 \\
\hline $\begin{array}{c}\text { Crude } \\
\text { root } \\
\text { extract }\end{array}$ & 100 & 0 & 37.08 & 75.42 & 115.50 & 114.48 \\
\cline { 2 - 7 } & 200 & 0 & 84.57 & 172.88 & 194.15 & 190.44 \\
\hline $\begin{array}{c}\text { Crude } \\
\text { stem } \\
\text { extract }\end{array}$ & 100 & 0 & 14.61 & 62.43 & 95.91 & 91.80 \\
\cline { 2 - 7 } & 200 & 0 & 57.02 & 127.12 & 144.15 & 152.46 \\
\hline $\begin{array}{c}\text { Crude } \\
\text { leaves } \\
\text { extract }\end{array}$ & 100 & 0 & 11.24 & 48.59 & 79.24 & 80.87 \\
\cline { 2 - 7 } & 200 & 0 & 48.60 & 101.41 & 128.36 & 120.77 \\
\hline
\end{tabular}

Table: 2 Analgesic activity of crude methanolic extract of S.kunthianus by hot plate method

\section{CONCLUSION}

Understanding and characterising the medicinal properties of herbal plants will help in the development of newer compounds and newer isolation techniques. This will help in the synthesis of active compounds from plants synthetically.

\section{REFERENCE}

[1] Balasubramaniam G, Sekar M, Ravi S, Badami S. Isolation and structural characterisation of Phytoconstituents from Strobilanthes kunthianus.Pharmacogn J.2020;12(6)suppl:1605-11

[2] Balasubramaniam G, Sekar M, Varadarajan M, Badami S. Analgesic and Anti-inflammatory Activities of Strobilanthes kunthianus in Experimental Animal Models.Research J. pharm. and tech.2020; 13(12):5844-5850

[3] Balasubramaniam G, Sekar M, Ravi S, Badami S. Pharmacognostical, Physicochemical and Phytochemical Evaluation of Strobilanthes kunthianus (Acanthaceae). Pharmacogn J.2020;12(4):731-41

[4] Bera S, Das S, Kalindi D. Botany, pharmacology and conservation status of wonder flower: Neelakurinji (Strobilanthes kunthianus(Nees)T. Anderson ex Benth).2020 